\title{
ARTICLE
}

\section{Neutron Spectra Measurements at JAEA MOX Fuel Facility}

\author{
Norio TSUJIMURA*, Tadayoshi YOSHIDA, Naoki SAGAWA, and Shigeru SHOJI \\ Nuclear Fuel Cycle Engineering Laboratories, Japan Atomic Energy Agency \\ 4-33, Muramatsu, Tokai-mura, Naka-gun, Ibaraki-ken, 319-1194, Japan
}

\begin{abstract}
The Nuclear Fuel Cycle Engineering Laboratories of the Japan Atomic Energy Agency fabricates MOX fuels for use in the Monju and Joyo FBRs at the Plutonium Fuel Production Facility (PFPF). The MOX pellet produced contains 20-30 wt \% of plutonium; therefore resulting neutron exposure in the fabrication process is a concern for radiation protection. To increase the neutron spectral knowledge, during the second reload-fuel production campaign for the Joyo reactor, neutron spectrum measurements were made at 20 locations representative of the PFPF using a Bonner multisphere spectrometer composed of a ${ }^{3} \mathrm{He}$ proportional counter and a set of four polyethylene spheres. The measurement results revealed the range of average neutron energies at all the locations to be from 0.35 to $1.6 \mathrm{MeV}$, and spectrum-averaged fluence-to-ambient dose equivalent conversion coefficients of 100 to $337 \mathrm{pSv} \mathrm{\textrm {cm } ^ { 2 }}$.
\end{abstract}

\section{KEYWORDS: MOX fuel, neutron spectrum, Bonner multisphere spectrometer, ambient dose equivalent}

\section{Introduction}

Nuclear fuel cycle technologies which maximize the utilization of nuclear fuels are essential to the Japan's nuclear energy strategy. In line with that the Nuclear Fuel Cycle Engineering Laboratories (NCL) of the Japan Atomic Energy Agency fabricates MOX (uranium and plutonium mixed oxide) fuel for use in the Joyo and Monju FBRs. The MOX pellet produced contains $20-30 \%$ of plutonium by weight, and emits neutrons from plutonium spontaneous fission and $(\alpha, n)$ reactions with oxygen. Increased use of plutonium could result in significant neutron exposure to workers engaged in the fabrication process, making therefore neutron dose measurements for radiation protection of great importance in nuclear fuel cycle.

It has long been recognized that the extensive knowledge on the neutron spectra in the workplaces is essential both in providing a technical basis for and in actually ascertaining the adequacy of the neutron dosimeters used ${ }^{1)}$. They also allow spectrum-dependent correction factors to be determined, which are of particular use with the thermoluminescent albedo dosemeters presently being used as personal dose-of-record dosemeters at $\mathrm{NCL}^{2)}$. For that reason neutron spectral measurements have been occasionally carried out in the MOX fuel facility work environments; for examples by Miyabe and Momose ${ }^{3)}$ in the late-1980s, and K. Yoshida et al. ${ }^{4}$ in the early 1990s. One of the present authors (N.T.) was also involved with precisely measuring the spectral fluence of neutrons directly emerging from cans containing MOX powders. ${ }^{5}$

During March 2007 through April 2008 the production campaign of second reload-fuel for the Joyo reactor took place. During that campaign neutron spectral measurements were made, after an interval of about 10 years, at several locations representative of the MOX fuel process line in the Plutonium Fuel Fabrication Facility (PFPF) of the NCL.

\footnotetext{
: Corresponding author, Tel.+81-29-282-1111, Fax.+81-29-282-

9534, E-mail; tsujimura.norio@jaea.go.jp
}

The main objectives of those measurements were to assess the current situations with neutron exposure and check whether any neutron spectral changes from the previous measurements had taken place. This paper presents the results of the latest neutron spectral measurement made at the MOX fuel facility.

\section{Materials and Methods \\ 1. Multisphere Spectrometer}

The neutron spectrometer used in the measurements was a Bonner Multisphere Spectrometer (BMS). The BMS is widely used in neutron spectrometry for radiation protection because of its high sensitivity, wide measurable energy range (thermal to $\sim 20 \mathrm{MeV}$ ), isotropic response and simplicity of operation.

The BMS employed was of the Uwamino and Nakamura design $^{6}$, which consists of a set of four polyethylene spheres of diameters $81,111,151$, and $231 \mathrm{~mm}$, and a central ${ }^{3} \mathrm{He}$ proportional counter with an external diameter of $5.1 \mathrm{~cm}$. The ${ }^{3} \mathrm{He}$ counter was manufactured by LND Inc. All the moderating spheres are covered with 0.5 -mm-thick cadmium shells to eliminate any incident thermal neutrons.

A special cart with NIM modules and drawers for moderators was constructed to facilitate transportation throughout the facility. During measurements the detector was positioned on a tripod at a height of $120 \mathrm{~cm}$ above the floor, which is the typical elevation of a personal dosemeter. The pulse signals from the ${ }^{3} \mathrm{He}$ counter were accumulated in a portable multichannel analyzer. Data was obtained by taking counts with a bare counter, a counter with a $\mathrm{Cd}$ shell, and a counter with moderators.

\section{Unfolding Procedure}

The unfolding code SAND-II ${ }^{7)}$ was used to determine the neutron spectrum from each set of count rate data. The basic principle of SAND-II is that it starts with a user-specified initial guess spectrum, calculates the responses of 5 detectors 
by folding the initial spectrum with detector response functions, improves the spectrum by comparing the calculated responses with the measured responses, and then iterates the process until a satisfactory spectrum is obtained.

The code uses a matrix of fluence responses (counts per unit neutron fluence), which was recalculated using a Monte Carlo computer code to update the old ANISN-based responses and verified through calibration measurements using a ${ }^{252} \mathrm{Cf}$ source of known strength and graphite-pilemoderated thermal neutrons. The code also requires an initial guess spectrum to start iteration. For an analysis of sensitivity of the unfolded spectrum to the initial spectrum, both previously-measured and calculated spectra were used as the initial spectrum. The latter spectra were obtained from combined calculations of SOURCES- $4 \mathrm{C}^{8)}$ and MCNP-4C ${ }^{9)}$. As examples of the initial spectra used, Fig. 1 plots the MOX source neutron spectrum calculated using SOURCES$4 \mathrm{C}$, and the neutron spectrum around the powder processing gloveboxes calculated using MCNP with almost the same configuration as the measurements.

From the past experience on the overall performance of a BMS of almost the same construction, the BMS used in the measurements could be expected to yield integral fluences and dose equivalents to within $\pm 10 \%$ of the reference values, provided that the initial spectrum was appropriate.

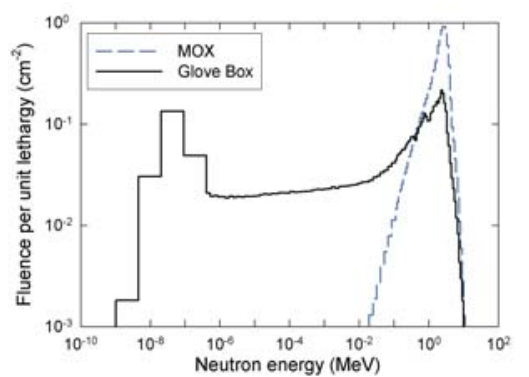

Fig. 1 Comparison of neutron spectral fluence, normalized to 1 $\mathrm{cm}^{-2}$, for a MOX source neutron spectrum computed using SOURCES-4C code (dashed line) and a spectrum around the powder processing glovebox calculated using MCNP code (solid line)

\section{Facility Description \\ 1. Outline}

The Plutonium Fuel Fabrication Facility (PFPF, a.k.a. Pu3 ) is a fully automated plant with a fabrication capacity sufficient to supply the MOX fuel for the Joyo and Monju FBRs. The PFPF commenced hot operation on October 1988, and to date has shipped a total of approx. 400 fuel assemblies, corresponding to $\sim 2.5$ tons of plutonium.

The MOX fabrication process is a direct application of the most common industrial uranium fuel fabrication process, except for the blending of the two materials of $\mathrm{UO}_{2}$ and $\mathrm{PuO}_{2}$. In the powder process the $\mathrm{UO}_{2}$ powder is mechanically blended with $(\mathrm{U}-50 \% \mathrm{Pu}) \mathrm{O}_{2}$ powder, latter of which is supplied from the finishing line of the Tokai Reprocessing Plant by means of microwave-heating denitration. The blended powder is fed to the pelletizing process, where it is pressed into green pellets, and then sintered in a furnace. In the rod process a cladding rod is loaded with pellets, and then sealed by TIG-welding. From the powder blending through to the rod welding all the processing equipment is housed in gloveboxes maintained at a lower pressure than the surrounding atmosphere. Bundles of fuel rods are then finally assembled into fuel assembly wrapper tubes.

A feature of the MOX fuel manufactured for use in FBRs is that it has the higher plutonium content of 20 to $30 \mathrm{wt} \%$ than that of several percent in MOX fuel typically used in LWRs; consequently giving rise to the potential for significant neutron exposure to workers.

\section{Measurement Locations}

Several locations within the PFPF were selected for the measurement. A total of 20 fields were characterized with the BMS, covering the entire work environment area involved with MOX fuel production. The selected locations included: (1) the powder blending and pelletizing process (13 points), (2) the assembling process (5 points), (3) the inspection process of the fuel assembly (1 point), and (4) around the fresh fuel transport packaging (1 point). From the radiation protection point of view the measurements were concentrated on the powder blending and pelletizing process area where neutrons at significant dose equivalent rates could be imparted. More detailed descriptions of the measurement locations are as follows, along with the measurement conditions.

(1) Powder blending and pelletizing: This process room has eight large gloveboxes housing the process equipment. The gloveboxes are two-sided airtight enclosures measuring $3 \mathrm{~m}$ wide $\times 3 \mathrm{~m}$ tall $\times 1 \mathrm{~m}$ deep. The gloveboxes are arranged in parallel lines and are interconnected with overhead trolley boxes through which the materials are moved to and from line to line via a buffer storage zone beyond a concrete partition. The gloveboxes are all constructed from stainless steel frames and 10-mm-thick acrylic resin panels. 35-mmthick slabs of lead-contained acrylic resin are also added to the faces of the gloveboxes to reduce the neutron exposure; however, this process line both currently and historically has provided the vast majority of personal neutron exposure cases, in particular with the glovebox operations for machinery maintenance. Much of that exposure originates from layers of plutonium oxide dust (referred to as 'holdup') on the internal surfaces of the gloves and the gloveboxes that unfavorably accumulates during the processes of ball-milling feed powders and grinding sintered pellets. The BMS was positioned at a distance of $30-50 \mathrm{~cm}$ from a face of the glovebox and at a height of $120 \mathrm{~cm}$ above the concrete floor, which is a typical arrangement for workers positioned at the face of a glovebox, and also closely matches the height of the open manipulation ports.

(2) Fuel Rod and Assembling: While the rod welding process is housed in smaller gloveboxes, all of the processes following rod welding are under a normal atmosphere. The equipment is maintained as 'clean', in contrast to the powder process, and therefore the processes are lightly shielded, but 
less exposure to workers occurs. The BMS measurements made here reflect the neutron spectrum for an observer standing near the process.

(3) Fuel Assembly Inspection: Measurements were made around a fuel assembly vertically supported by the inspection tower. A higher energy spectrum was expected due to there being no shielding and less room-scattering in an open large-size room.

(4) Fresh Fuel Transport Packaging: For the delivery of fresh fuel assemblies to Joyo, dedicated transport packaging incorporating the neutron shielding is used. The packaging is approximately $3 \mathrm{~m}$ long and $0.4 \mathrm{~m}$ in diameter with impact limiters on both ends. The packaging was horizontally situated on the concrete floor, thereby allowing the measurement to reflect the neutron spectrum an observer standing near the packaging would be exposed to.

Photographs of a representative measurement set-up are given in Fig. 2. At each of the locations, the time duration of each measurement was 180-300 s, with the total time to complete all measurements at each field being about $30 \mathrm{~min}$. The statistical errors were typically 1-3\%.

\section{Results and Discussion \\ 1. Neutron Spectra}

Figure 3 provides plots of the unfolded spectral fluence in log energy intervals at four representative measurement points: (a) a glovebox for ball-milling in the powder blending process (position 4); (b) a contamination check apparatus for the welded fuel rods in the assembling process (position 16); (c) the fuel assembly (position 19); and (d) the fuel transport packaging (position 20). The integrated fluence over the energy was set to unity for the sake of comparison. Slightly wide variations in the neutron spectrum can be seen that can be explained by the different shielding and room geometries. Spectrum (a) is the softest, typically encountered around the gloveboxes, and is composed of a fast component directly originating from the source spectrum plus thermal/intermediate components attributed to by the hydrogenous-shield moderation and room reflections. The hardest spectrum of (c) is found around the fuel assembly, where there is essentially no shielding. The PFPF field neutron spectra are similar to those at other similar facilities ${ }^{10)}$

Figure 4 provides plots of dose equivalent distributions, which were directly converted from the four spectral fluences of Fig. 3 using ICRP 74 fluence-to-ambient dose equivalent conversion coefficients. The differences among the spectra are masked by the great importance of the conversion coefficients being biased to the high energy region. The dose equivalent distributions reveal the fact that the majority of neutron dose equivalents is attributed to fast neutrons $>100 \mathrm{keV}$, regardless of the measuring positions in the PFPF.

\section{Spectrometric and Dosimetric Quantities}

Table 1 summarizes the spectrometric and dosimetric quantities for the neutron spectrum at all the measurement positions. The range of the average neutron energies was from 0.35 to $1.6 \mathrm{MeV}$, and spectrum-averaged fluence-to-
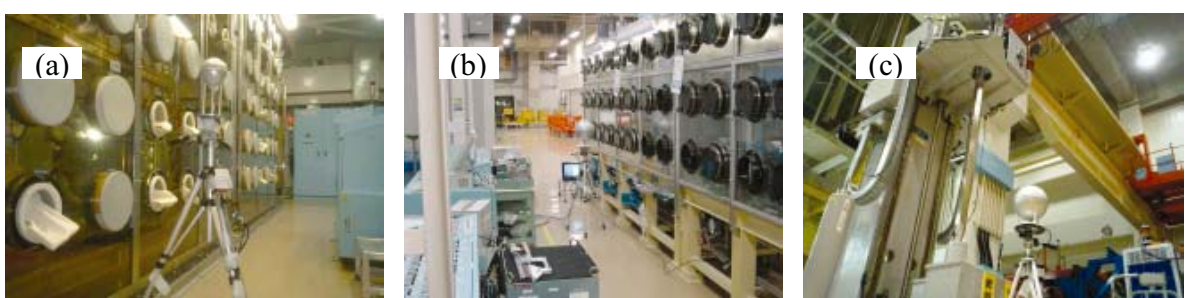

Fig. 2 Photographs of a representative measurement set-up for (a) the large glovebox for ball-milling in powder processing process (position 4), (b) the glovebox housing the contamination check apparatus of the welded fuel rods (position 16), and (c) the fuel assembly (position 19)
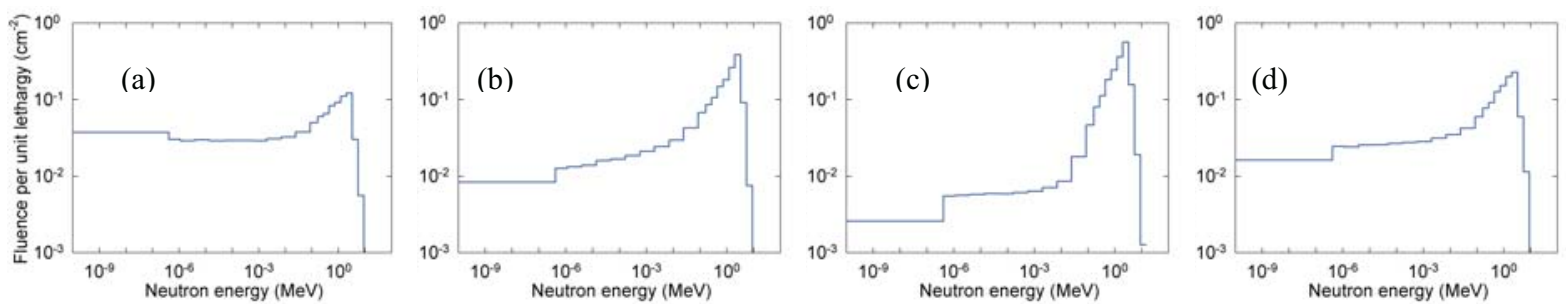

Fig. 3 Measured neutron spectral fluence for the measurement locations of (a) to (d). See text for explanatory information.
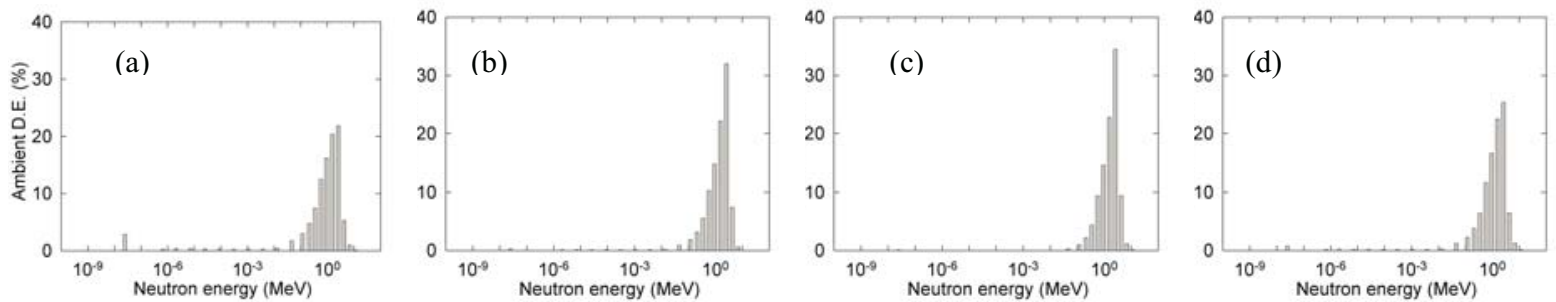

Fig. 4 Ambient dose equivalent distribution for the measurement locations of (a) to (d). See text for explanatory information. 
Table 1 Summary of BMS measurements: fluence-average neutron energy $\left(E_{\phi}\right)$, spectrum-average fluence-to-ambient dose equivalent conversion coefficients $\left(h^{*}\right)$ and their relative contributions in \%

\begin{tabular}{|c|c|c|c|c|c|c|}
\hline \multirow[t]{2}{*}{ Position $^{a}$} & \multirow[t]{2}{*}{ Process $^{b}$} & \multirow{2}{*}{$\begin{array}{c}E_{\phi} \\
(\mathrm{MeV})\end{array}$} & \multirow{2}{*}{ 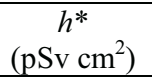 } & \multicolumn{3}{|c|}{ Relative fluence (ambient dose equivalent) in \% } \\
\hline & & & & $>0.74 \mathrm{MeV}$ & $0.74 \mathrm{MeV}-0.4 \mathrm{eV}$ & Thermal \\
\hline 1 & PP & 0.53 & 139 & $22(67)$ & $54(31)$ & $24(2)$ \\
\hline 2 & PP & 0.53 & 139 & $23(68)$ & $50(30)$ & $27(2)$ \\
\hline 3 & PP & 0.35 & 100 & $15(62)$ & $50(34)$ & $36(4)$ \\
\hline 4 & PP & 0.42 & 115 & $18(65)$ & $51(32)$ & $31(3)$ \\
\hline 5 & PP & 0.46 & 128 & $20(64)$ & $54(34)$ & $26(2)$ \\
\hline 6 & PP & 0.45 & 117 & $19(66)$ & $49(31)$ & $33(3)$ \\
\hline 7 & PP & 0.64 & 161 & $26(68)$ & $52(31)$ & $21(1)$ \\
\hline 8 & PP & 0.35 & 100 & $15(61)$ & $49(35)$ & $37(4)$ \\
\hline 9 & $\mathrm{PP}$ & 0.40 & 107 & $16(63)$ & $53(34)$ & $30(3)$ \\
\hline 10 & PP & 0.54 & 144 & $22(64)$ & $61(35)$ & $17(1)$ \\
\hline 11 & PP & 0.63 & 158 & $27(70)$ & $50(28)$ & $24(2)$ \\
\hline 12 & PP & 0.75 & 185 & $31(70)$ & $54(30)$ & $15(1)$ \\
\hline 13 & $\mathrm{PP}$ & 0.59 & 154 & $25(68)$ & $56(30)$ & $19(1)$ \\
\hline 14 & AS & 1.1 & 257 & $47(77)$ & $49(23)$ & $4(0)$ \\
\hline 15 & AS & 0.72 & 164 & $30(76)$ & $54(23)$ & $15(1)$ \\
\hline 16 & AS & 1.1 & 249 & $46(77)$ & $47(23)$ & $7(0)$ \\
\hline 17 & AS & 1.0 & 229 & $42(76)$ & $49(23)$ & $9(1)$ \\
\hline 18 & AS & 0.78 & 201 & $33(68)$ & $58(31)$ & $9(1)$ \\
\hline 19 & FA & 1.6 & 337 & $67(83)$ & $31(17)$ & $2(0)$ \\
\hline 20 & $\mathrm{TP}$ & 0.76 & 186 & $32(72)$ & $54(27)$ & $13(1)$ \\
\hline $\begin{array}{c}1992-1995 \\
\text { measurements }\end{array}$ & PP & $0.45-0.75$ & $110-168$ & $\begin{array}{c}18-30 \\
(69-75)\end{array}$ & $\begin{array}{c}39-50 \\
(23-28)\end{array}$ & $\begin{array}{c}22-41 \\
(1-4)\end{array}$ \\
\hline
\end{tabular}

${ }^{a}$ Positions are in ascending order of the process.

${ }^{\mathrm{b}} \mathrm{PP}$ : Powder blending and Pelletizing process, AS: Assembling process, FA: Fuel Assembly inspection, TP: Transport Packaging.

ambient dose equivalent conversion coefficients of 100 to $337 \mathrm{pSv} \mathrm{cm}^{2}$. Although thermal neutrons accounted for $\sim 30 \%$ of the total neutron fluences measured at the glovebox lines, they only contribute at most $\sim 4 \%$ of the total neutron dose equivalents. Neutrons with energies above $\sim 700 \mathrm{keV}$, which corresponds to a threshold energy value of a certain commercially available electronic personal neutron dosemeter ${ }^{11)}$, account for $60-80 \%$ of total dose equivalent.

The data given at the foot of Table 1 was measured in the early 1990s at similar, but not identical, work locations in the PFPF. Comparing them reveals no significant changes in the neutron spectrum in the work environments.

\section{Conclusions}

For a good knowledge on neutron spectra in the work environments of the Plutonium Fuel Production Facility of the JAEA, neutron spectrum measurements using a multisphere spectrometer were made at 20 representative MOX fuel fabrication process locations. The neutron spectrometric and dosimetric features were wellcharacterized. The measurement results will contribute to improving current radiation protection programs at JAEA MOX fuel facilities.

The neutron spectral fluence data is also available from the head author (N. Tsujimura) as a Microsoft Excel spreadsheet.

\section{Reference}

1) ICRU, Determination of operational dose equivalent quantities for neutrons. ICRU Report 66 (2001).
2) N. Tsujimura, K. Kanai, T. Momose, "Thermoluminescence Neutron Dosimetry in the JNC MOX Fuel Fabrication Facilities,” J. Nucl. Sci. Tech. Suppl. 4, 203-206 (2004).

3) A. Yamato, K. Miyabe, T. Momose, et al., "Development of the Neutron Energy estimation Method and Study of Neutron Dosimetry in Nuclear Fuel Facilities," PNC Technical Review, 65, 125-18, (1988), [in Japanese].

4) K. Yoshida, personal communication, (2001).

5) N. Nakao, N. Tsujimura, T. Nakamura, et al., "Measurement of Neutron Energy Spectra of $\mathrm{PuO}_{2}-\mathrm{UO}_{2}$ Mixed Oxide Fuel and Penetrated through Surrounding Lead-Acryl Shield," J. At. Energy Soc. Japan, 35, 1103-1114 (1993), [in Japanese].

6) Y. Uwamino, T. Nakamura, "Two types of Multi-Moderator Neutron Spectrometers: Gamma-ray Insensitive Type and HighEfficiency Type," Nucl. Instrum. Methods, A239, 299-309 (1985).

7) W. N. McElroy, S. Berg, T. Crockett et al., Spectra Unfolding, AFWL-TR-67-41, Vol.1-4, Air Force Weapons Laboratory, (1967).

8) E. F. Shores, SOURCES 4C: A code system for calculating $(\alpha, n)$, spontaneous fission, and delayed neutron sources and spectra, LA-UR-02-1839, Los Alamos National Laboratory, (2002)

9) J. F. Briesmeister, ed., MCNP - A general Monte Carlo Nparticle transport code, version 4C, LA-13709-M, Los Alamos National Laboratory, (2000).

10) R. Olaerts, P. Kockerols, A. Renard, et al., "Determination of Neutron Spectra in a MOX Plant for the Qualification of the BPPND Bubble Detector," Health Phys. 77, 200-206 (1999).

11) N. Tsujimura, Y. Yoshida, C. Takada, et al., "Performance test of the electronic personal neutron dosemeter in neutron fields simulating workplaces of MOX fuel fabrication facilities," Proc. 12th Int. Congress of Int. Radiat. Prot. Asso. (IRPA12), Oct. 1924, 2008, Buenos Aires, Argentina, (2008). [CD-ROM] 